\title{
Lyophilisate for Oculonasal Suspension/Use in Drinking Water Dosage
} Form

National Cancer Institute

\section{Source}

National Cancer Institute. Lyophilisate for Oculonasal Suspension/Use in Drinking Water

Dosage Form. NCl Thesaurus. Code C149638.

Solid preparation for veterinary use consisting of a freeze-dried powder intended to be dispersed in the specified liquid to create a suspension for oculonasal use, or to be dispersed in the animal drinking water. 